\title{
Dynamic Microwave Photonic Filter Using Separate Carrier Tuning Based on Stimulated Brillouin Scattering in Fibers
}

\author{
Juan Sancho, Sanghoon Chin, Mikel Sagues, Member, IEEE, Alayn Loayssa, Member, IEEE, Juan Lloret, \\ Ivana Gasulla, Salvador Sales, Senior Member, IEEE, Luc Thévenaz, and José Capmany, Fellow, IEEE
}

\begin{abstract}
Dynamic reconfiguration of a microwave photonic filter by tuning its basic delay based on stimulated Brillouin scattering-induced slow light and optical phase shift of the optical carrier is experimentally implemented. The measurements confirm that the free spectral range of the filter changes when a Brillouin pump is applied. These results demonstrate the potential of the separate carrier technique in microwave photonics applications.
\end{abstract}

Index Terms-Microwave optical filters, microwave photonics, optical delay line, optical signal processing, slow light.

\section{INTRODUCTION}

$\mathbf{T}$ HE development of tunable photonic delay lines is of paramount importance in broadband digital and analog communication systems. Recently, as a promising approach to provide all-optically controlled signal delaying, slow light (SL) techniques have been extensively investigated in various materials based on a number of physical mechanisms [1], [2]. However, it was soon identified that any slow light linear system based on complex optical resonances suffers from a critical deadlock in terms of optical storage capacity. As already discussed in previous works [3], [4], the maximum achievable time delay that signal pulses can experience is strictly restricted up to a few periods of the signal. On the contrary, slow light can be implemented as a robust solution for all-optical analogue signal processing [5], [6]. In microwave photonics, tunable optical delay-lines are particularly required to implement microwave photonic filters (MPFs) and to support phased array antenna (PAA) systems. These past few years, MPFs have been widely developed as a result of the considerable extension of

Manuscript received March 26, 2010; revised September 16, 2010; accepted September 26, 2010. Date of publication September 30, 2010; date of current version November 17, 2010. This work was supported by the European Union FP7 project GOSPEL, and by the Generalitat Valenciana PROMETEO 2008-092 Microwave Photonics excellency research project, Plan Nacional I+D T EC2007-68065-C03-01 and TEC2007-67987-C02-02.

J. Sancho, J. Lloret, I. Gasulla, S. Sales, and J. Capmany are with the iTEAM Research Institute, Universidad Politécnica de Valencia, 46022 Valencia, Spain (e-mail: juasandu@iteam.upv.es; jualloso@iteam.upv.es; ivgames@iteam.upv.es; ssales@dcom.upv.es; jcapmany@dcom.upv.es).

S. Chin and L. Thévenaz are with Ecole Polytechnique Fédérale de Lausanne, Institute of Electrical Engineering, 1015 Lausanne, Switzerland (e-mail: sanghoon.chin@epfl.ch; luc.thevenaz@epfl.ch).

M. Sagues and A. Loayssa are with the Department of Electrical and Electronic Engineering, Universidad Pública de Navarra, 31006 Pamplona, Spain (e-mail: mikel.sagues@unavarra.es; alayn.loayssa@unavarra.es).

Color versions of one or more of the figures in this letter are available online at http://ieeexplore.ieee.org.

Digital Object Identifier 10.1109/LPT.2010.2082514 their potential applications and also their inherent advantages compared to traditional radio-frequency (RF) circuits [7]. However, these schemes still require novel solutions to overcome the positive-valued nature of the coefficients that could be implemented under incoherent operation. Actually, this restriction limits the domain of achievable filter transfer functions. Several methods to realize microwave filters with negative coefficient and complex-valued coefficient have been already proposed, so as to overcome this limitation and to enhance the frequency tuning range of the filter [5]-[7]. Nevertheless, most MPFs rely on discrete-time signal processing using real valued coefficients, which requires the use of tunable optical true time delays (TTDs).

In practice, the use of slow light-induced tunable delay for the realization of microwave filters has been limited since the product between the maximum achievable group delay and the signal bandwidth is essentially constant. Thus only modest group delays can be achieved for broadband baseband signals, as already observed in digital signal delaying. In most practical microwave photonic systems, however, the RF subcarrier frequency is much higher than the bandwidth of the conveyed signal. Therefore, no information is carried in the broad frequency spacing between the optical carrier and the single-sideband microwave envelope. For this particular context an elegant solution has been theoretically proposed to overcome this problem by separate carrier tuning (SCT) the optical phase of the carrier [8]. Thus, the requirement of constant group (time) delay across the entire bandwidth can potentially be relaxed across this region as long as an appropriate phase-shift is also imparted to the carrier. The experimental demonstration of the SCT technique is yet to be provided and is of paramount importance in order to show the feasibility of implementing TTDs using slow light principles based on narrowband resonant media, in particular in fiber based stimulated Brillouin scattering (SBS) amplifiers and integrated coupled resonator optical waveguides.

In this letter, we provide this demonstration using SBS-induced slow light and phase-shifting of the optical carrier with a fiber Bragg grating. To the best of our knowledge, this is the first experimental report of the SCT concept in the field of microwave photonics.

\section{Microwave Photonic FiLter Implementation USING SCT}

The basic configuration of a notch-type incoherent microwave photonic filter is a two-tap structure, which yields a 


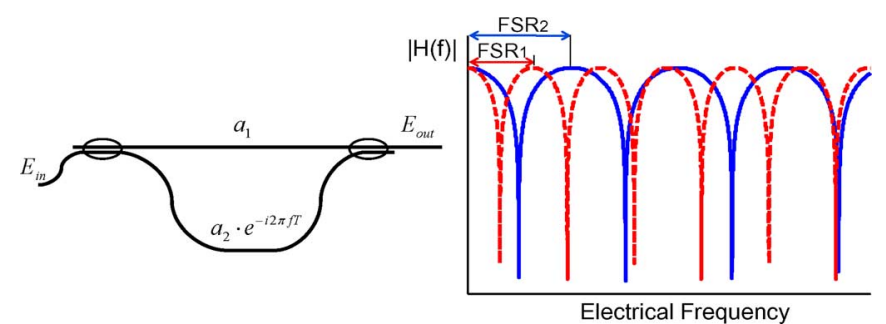

Fig. 1. Layout and transfer function of a two-tap microwave photonic filter.

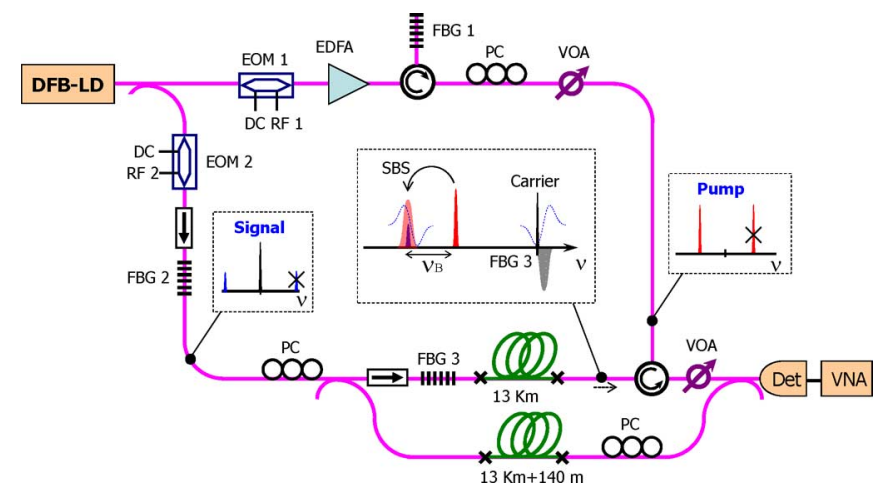

Fig. 2. Experimental setup.

periodic spectral response. Fig. 1 shows this Mach-Zehnder layout and its frequency response. The transfer function of this structure is given by [5]

$$
H(f)=a_{1}+a_{2} \cdot e^{-i(2 \pi f T)}
$$

where $a_{1}$ and $a_{2}$ are the filter taps coefficients that can be real or complex, and $T$ is the filter's basic delay. The notch depth depends on the amplitude difference between these coefficients while the response period is directly related to the path delay imbalance on one arm with respect to the other, free spectral range $(\mathrm{FSR})=1 / T$. Therefore, the tuning of the FSR of a transversal filter requires the variation of the basic delay.

Fig. 2 schematically depicts a two-tap SBS tunable optical filter. It consists of two main building blocks to reconfigure the frequency response of the MPF. The upper part of the diagram is dedicated to prepare and deliver the pump required for the implementation of the SBS-based tunable delay and the lower part performs the tunable MPF.

A distributed-feedback (DFB) laser operating at $1550 \mathrm{~nm}$ was used as a light source and its output was split using a 3-dB fiber-optic coupler. One branch was modulated after passing through an external electro-optic modulator (EOM1) with a tunable modulation frequency from 0 to $20 \mathrm{GHz}$. A direct current (dc) bias applied to the EOM1 was properly adjusted in order to suppress the carrier at the output of the modulator. Then it was delivered to the fiber Bragg grating (FBG1) via a circulator to precisely transmit the lower sideband showing more than 20-dB suppression ratio between the two sidebands. The output signal then amplified by an erbium-doped fiber amplifier to be used as a controlled pump for the Brillouin gain that generates the tunable delay. Its output power was controlled by a variable optical attenuator (VOA) for the optical control of generated tunable delay. In the other branch, the signal from the DFB laser feeds the EOM2 which is driven by the RF signals that were going to be optically processed. Another fiber Bragg grating (FBG2) was used to remove the upper sideband. Then, it was launched into the MPF [7] that operates under an incoherent regime. A circulator was used to counterpropagate the SBS pump signal with the optical single-sideband-modulated carrier in one of the two branches of the MPF structure. The inset in Fig. 2 illustrates the spectral position of the Brillouin gain resonance generated by the pump, inducing a linear phase shift in the vicinity of the microwave modulation frequencies. Therefore, the frequency of the RF signal modulating the EOM2 should be shifted by $\nu_{B}$ (the Brillouin frequency) as compared to the frequency of the RF signal modulating the EOM1. Meanwhile, the optical carrier has to be phase tuned [8] using the phase spectrum response of a third grating (FBG3). Finally, the outputs from the two branches of the MPF are combined in the output coupler and the MPF response, (1), is measured with a vector network analyzer (VNA). A 140-m-long optical fiber was introduced in one branch of the MPF layout to provide the desired frequency response. In addition, the imbalance in optical path length between the two branches provides not only the condition of incoherent regime, but also, offers the possibility to study the feasibility of the SCT technique by looking at several resonances of the MWF. Polarization controllers (PC) have been used to maximize the SBS effect. The complexity of the experimental setup is due to two main reasons. First, the resonant mechanism providing SFL is based on SBS, which requires a pumping path (the setup should be simpler in the case of a passive coupled resonator). Second, it is assembled to implement a two tap filter where the SBS-based SFL can operate either as a phase shifter or a TTD. It is, therefore, an ad-hoc (i.e., not optimized) configuration assembled for the purpose of demonstrate (and distinguish) both operations.

\section{EXPERIMENTAL VALIDATION}

A 13-km dispersion-shifted fiber (DSF) was used as Brillouin gain medium; the Brillouin frequency shift and the resonance bandwidth were measured to be $10.7 \mathrm{GHz}$ and $40 \mathrm{MHz}$, respectively. The microwave signal was generated at $30.7 \mathrm{GHz}$ through EOM2 and an RF tone of $20 \mathrm{GHz}$ was applied to EOM1 to match the Brillouin frequency of the fiber. Fig. 3(a) shows the spectral profile of the SBS gain resonance while the microwave subcarrier frequency was swept in the vicinity of the resonance and also the corresponding phase shift of the microwave signal with respect to the microwave signal frequency. On the other hand, Fig. 3(b) shows FBG3 gain and phase response, too. The inset in Fig. 3(a) shows the SBS gain and phase shift within a $10-\mathrm{MHz}$ range. There is a linear phase change and also a moderate change in the gain. It is very important for the MPF, to control both the TTD as well as the amplitude coefficients of the filter, i.e., $a_{1}$ or $a_{2}$. To implement the TTD, a linear phase slope is required, since $T=1 /(2 \pi) d \phi / d f$. Then, the maximum achievable TTD value over the $10-\mathrm{MHz}$ span is $51.4 \mathrm{~ns}$. This delay can be tuned by changing the optical power of the pump signal.

To check the validity of the SCT technique, we first measured the implementation of phase shift in the optical carrier by recording the filter spectral transfer function which in this case corresponds to a complex-valued two-coefficient MPF [5]-[7]. Fig. 4(a) shows the shift of spectral response of the MPF, preserving, as expected, its free spectral range and its spectral shape. This effect confirms that the phase shift impressed in the optical carrier propagating through the upper branch of the 


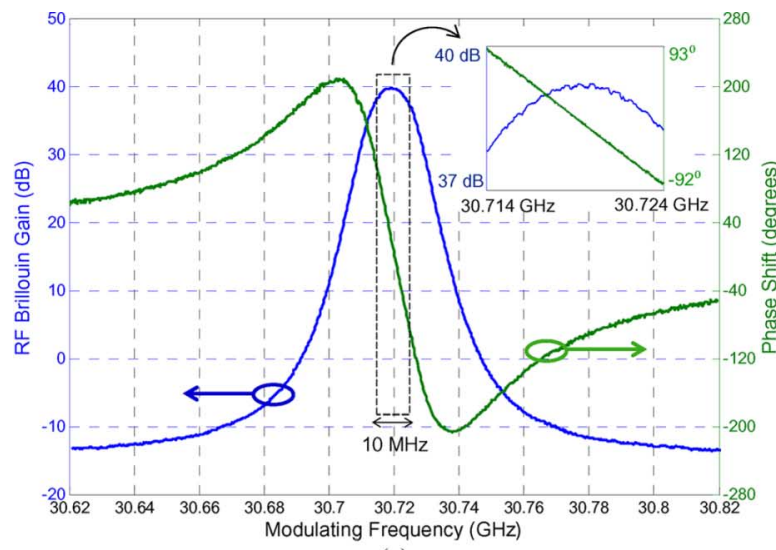

(a)

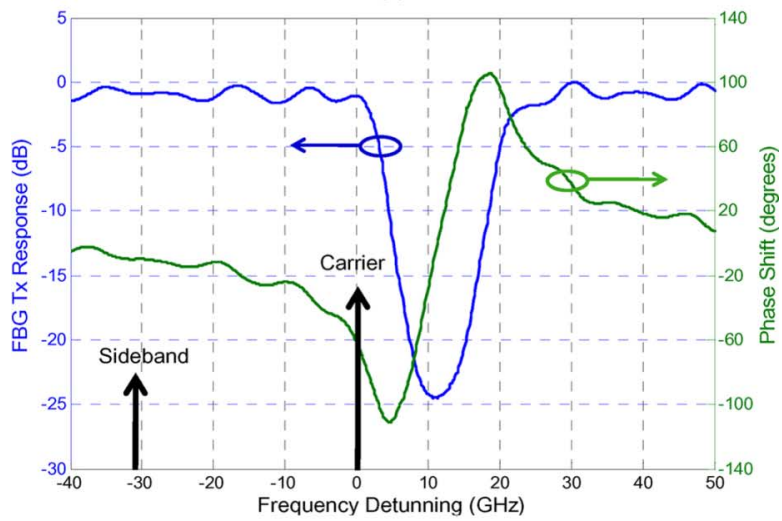

(b)

Fig. 3. (a) SBS and (b) FBG3 gain and phase shift measurements.

MPF is changed by tuning the central frequency of FBG3. Two different phase shifts have been displayed with a difference of almost $180^{\circ}$. The two branches of the MPF have a length imbalance of $140 \mathrm{~m}$ (700 ns), which led to an FSR of $1.43 \mathrm{MHz}$.

The SCT predicts that a TTD can be created, which should imply a different behavior of the MPF since its FSR has to change upon tuning the delay. Fig. 4(b) confirms that the FSR of the filter changes when the pump is applied. If a $16-\mathrm{dBm}$ pump power is counterpropagated into the upper branch of the MPF, the SBS generates a TTD around 51.4 ns (see Fig. 3). This implies that the FSR should be increased up to $1.54 \mathrm{MHz}$ [see Fig. 4(b)], showing an excellent agreement with theoretical prediction from (1). FBG3 is used for the carrier phase adjustment as required by the SCT technique [8]. The phase of the microwave signal can be described as

$$
\varphi\left(w_{\mathrm{RF}}\right)=\phi\left(\omega_{c}\right)-\phi\left(\omega_{s}\right)+\left.w_{\mathrm{RF}} \frac{\partial \phi\left(\omega_{c}\right)}{\partial w}\right|_{\omega_{c}-\omega_{s}}
$$

where $\phi\left(\omega_{c}\right)$ and $\phi\left(\omega_{s}\right)$ are the phase shifts at the carrier and sideband frequency, respectively. For TTD, $-60^{\circ}$ of carrier compensation is needed in our particular case. Fig. 4 shows an excellent agreement of the theoretical and experimental results.

\section{CONCLUSION}

We have experimentally demonstrated, for the first time to our knowledge, the feasibility of implementing TTDs using slow

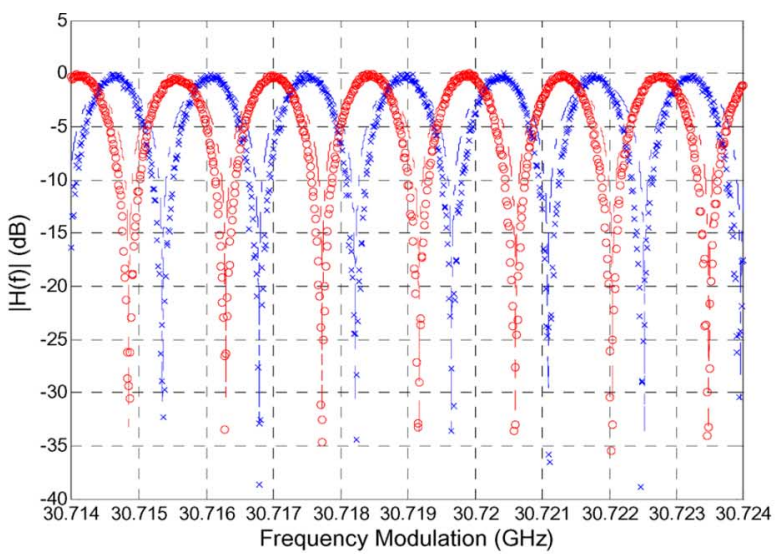

(a)

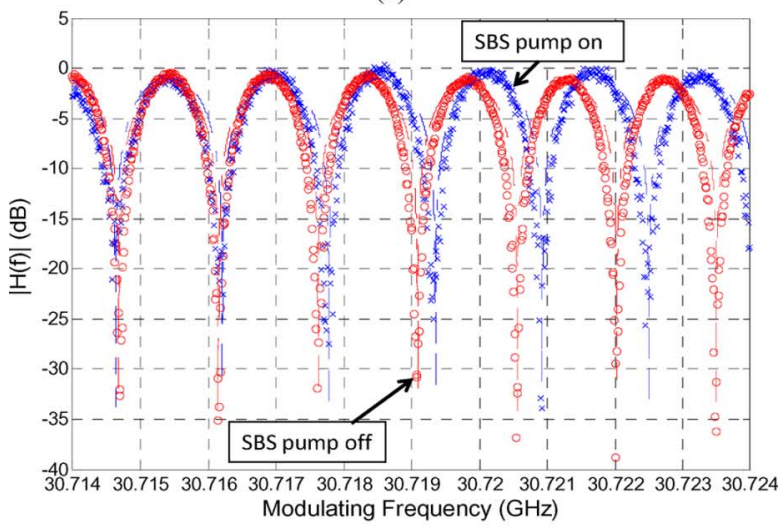

(b)

Fig. 4. Measured (circles and crosses) and simulated (dashed lines) filter frequency response (a) tuning the FBG3 position and (b) for 16-dBm SBS pump power and for no pump.

light based on a narrowband resonance by means of the SCT technique. A two-tap microwave photonic filter where SFL is implemented using SBS has been employed for this purpose.

The effect of TTD induced by the SCT method was clearly verified as the FSR of the filter was varied. We believe that the SCT technique can be a competitive solution for microwave photonics applications.

\section{REFERENCES}

[1] R. W. Boyd and D. J. Gauthier, "Slow and fast light," Prog. Opt., vol. 43, no. 6, pp. 497-530, 2002.

[2] J. B. Khurgin and R. S. Tucker, Slow Light: Science and Applications. Boca Raton, FL: CRC Press, 2009.

[3] K. Y. Song, M. Gonzalez-Herráez, and L. Thévenaz, "Observation of pulse delaying and advancement in optical fibers using stimulated Brillouin scattering," Opt. Express, vol. 13, pp. 82-88, Jan. 2005.

[4] Y. Okawachi, M. S. Bigelow, J. E. Sharping, Z. Zhu, A. Schweinsberg, D. J. Gauthier, R. W. Boyd, and A. L. Gaeta, "Tunable all-optical delays via Brillouin slow light in an optical fiber," Phys. Rev. Lett., vol. 94, no. 15, p. 153902, Apr. 2005.

[5] M. Sagues, R. Garcia, A. Loayssa, S. Sales, and J. Capmany, "Multi-tap complex-coefficient incoherent microwave photonic filters based on optical single-sideband modulation and narrow band optical filtering," Opt. Express, vol. 16, no. 1, pp. 295-303, Jan. 2008.

[6] W. Xue, S. Sales, J. Mork, and J. Capmany, "Widely tunable microwave photonic notch filter based on slow and fast light effects," IEEE Photon. Technol. Lett., vol. 21, no. 3, pp. 167-169, Feb. 1, 2009.

[7] J. Capmany and D. Novak, "Microwave photonics combines two worlds," Nature Photon., vol. 1, no. 6, pp. 319-330, Jun. 2007.

[8] P. A. Morton and J. B. Khurgin, "Microwave photonic delay line with separate tuning of the optical carrier," IEEE Photon. Technol. Lett., vol. 21, no. 22, pp. 1686-1688, Nov. 15, 2009. 